\title{
Some remarks on definition, resolution and contrast in photothermal imaging
}

bY H.G. WALTHER and U. SEIDEL

Institut für Optik und Quantenelektronik, Physikalisch-Astronomische Fakultät, Friedrich-Schiller-Universität Jena, Max-Wien-Platz 1, O-6900 Jena, Germany

\begin{abstract}
Photothermal imaging can be described quantitatively by means of a point spread function (PSF), which represents the image of a buried thermal point defect. From this PSF conclusions can be drawn about amplitude and phase contrast, halfwidth and resolution of photothermal imaging. Radiometric and mirage measurements from an appropriately designed model sample confirm the theoretical results.
\end{abstract}

\section{Nomenclature}

$S \quad$ - photothermal signal

$G \quad$ - photothermal point spread function

$k \quad$ - thermal conductivity

Qc - thermal density

A - defect strength with respect to $k$

$B \quad$ - defect strength with respect to $\varrho c$

$i \quad$ - imaginary unit $(-1)^{1 / 2}$

$\mu \quad$ - thermal diffusion length

$F \quad$ - defect distribution

$W \quad$ - heating beam profile

$K \quad$ - contrast function

$\Phi \quad$ - phase of contrast function

$z_{0} \quad$ - defect depth

$x_{0} \quad$ - lateral distance betwen excitation beam and defect

$R \quad$ - heating beam radius

\section{Introduction}

Thermal waves provide a useful tool for the investigation of inhomogeneities of non optical properties in solids as shown, for instance, by BUSSE and ROSENCWAIG [1] twelve years ago. Recently photothermal measuring techniques have been used successfully to detect hidden patterns inside of opaque and strongly scattering samples [2]. The field of this so-called photothermal imaging includes non destructive evaluation of materials and components with pattern sizes in the $\mathrm{mm}$-range as well as photothermal imaging with sub- $\mu \mathrm{m}$ resolution. The aim of photothermal imaging techniques consists in obtaining informations about the location (especially the depth) of optically invisible inhomogeneities within the sample, about the shape of these defects and about the strength or - in other words - the amount of deviation from the mean values caused by inhomogeneities. We can get this information if we know the definition and contrast of the photothermal imaging technique used.

The exact description of the photothermal signal formation requires the consideration of the thermal wave generation, their propagation within the sample and their scattering from 


\section{http://dx.doi.org/10.21611/qirt.1992.039}

thermal inhomogeneities. A general recipe how to perform such calculations was given by a recent paper of MANDELIS [3].

In this paper we will use the concept of the photothermal transfer function. Cornparing photothermal and optical imaging we will introduce a photothermal point spread function PSF. This PSF describes the photothermal signal distribution generated by a hidden point-like defect. From the PSF we can draw conclusions about the halfwidth of the point imace and we will be able to predict the conditions of the highest measuring contrast.

\section{Photothermal point spread function}

The quality of the photothermal imaging can be evaluated by its definition, resolving power and contrast [ 4 and 5 ]. When studying these problems it is advantageous to use the formalism of point spread function (PSF). This function descended from optical microscopy [6] describes the blurring of the image of a point object as the result of the physical mechanism of image formation. In the case of photothermics the scattering of heavily damped thermal waves from thermal inhomogeneities makes it possible to image such structures.

The principle of the photothermal imaging process is sketched in figure 1 . We restrict our investigations to the case of periodically modulated laser light, which is tightly focussed at the strongly surface absorbing sample. For the consideration of weakly absorbing samples we have to take into account subsurface heating which will be reported in a forthcoming paper [7]. We discuss such photothermal techniques which yield a signal proportional to the averaged surface temperature oscillation. This condition is met when detectors with sufficiently large aperture will be used.

Now we consider a point-like thermal inhomogeneity. The thermal waves will be scattered and will modify the surface temperature distribution. Therefore the resulting signal consists of $S_{o}$ and a deviation $S_{1}$, which depends on lateral and vertical distance between heating beam and point defect. In the case of a point-like defect, $S_{1} / S_{0}$ is identical with the PSF of photothermal imaging which is called $G$. To calculate the PSF we have to solve the heat diffusion equation with spatially dependent coefficients. If the deviation of them from the thermal mean values is small enough we succeed in performing a perturbation theoretical approach [12 and 13]. With the thermal defect strengths $A=\Delta k / k_{0} B=\Delta(\varrho \cdot c) /(\varrho \cdot c)_{o}$ we finally get the PSF of a surface absorber.

$$
G(r)=\frac{\pi}{2} \sigma^{2} \frac{1}{\sqrt{r^{2}+z_{0}^{2}}} \exp \left(-\sigma\left(z_{0}+\sqrt{r^{2}+z_{0}^{2}}\right)\right\}\left[\frac{A z_{0}}{\sqrt{r^{2}+z_{0}^{2}}}\left(1+\frac{1}{\sigma \sqrt{r^{2}+z_{0}^{2}}}\right)+B\right]
$$

with $\sigma^{2}=2 i / \mu^{2}, \mu$ is the thermal diffusion length of the homogeneous material, $r$ and $z_{0}$ are lateral and vertical offset between point defect and heating beam. A more detailed discussion of the PSF is given in [8]. When we scan an extended inhomogeneity of shape $F(x, y)$ localized at depth $z_{o}$ below the surface by heating laser profile $W(x, y)$, then the signal deviation $S_{d e v}$ from the unperturbated signal $S_{0}$ is given by the multiple convolution integral

$$
S_{d e v}=S_{0}[W * * F * G]
$$

\section{Photothermal contrast functions}

If the sample under test is scanned photothermally we usually record signal amplitude and/or phase by means of a vector lock-in amplifier. The detectability of any structure-related signal in relation to the background signal of the homogeneous material is given by the contrast function $K$ which can be defined for both amplitude and phase (ph): 


$$
\begin{aligned}
& K_{A}=\frac{\left|S_{0}+S_{d e v}\right|-|S|_{0}}{\left|S_{0}\right|} \\
& K_{\Phi}=p h\left(S_{0}+S_{d e v}\right)-p h\left(S_{0}\right)
\end{aligned}
$$

These definitions are appropriate for all measuring techniques where a photothermal signal is obtained at each measuring point. With $S_{o}=\left|S_{o}\right| \exp \left(i \Phi_{0}\right), S_{d e v}=\left|S_{d e v}\right| \exp \left(i \Phi_{d e v}\right)$, where $|S|$ and $\Phi$ are signal amplitude and phase, respectively and with the presumption $\left|S_{1}\right|$. $\left|S_{d e v}\right|<<\left|S_{o}\right|$ which is reasonable for weak thermal distortions one obtains the contrast functions for the buried point defect. We find that these functions are connected with the complex valued PSF:

$$
\begin{aligned}
& K_{A} \approx \frac{\left|S_{1}\right|}{\left|S_{0}\right|} \cos \left(\Phi_{1}-\Phi_{0}\right)=\Re e(G) \\
& K_{\Phi} \approx \frac{\left|S_{1}\right|}{\left|S_{0}\right|} \sin \left(\Phi_{1}-\Phi_{0}\right)=\Im m(G)
\end{aligned}
$$

With the known $G$ we can calculate the experimentally expected amplitude and phase contrast when scanning the sample over a buried point defect. For extended thermal defects the contrast can be estimated replacing $G$ by the corresponding expression $\left(W^{*}{ }^{*} F^{* *} G\right)$ of equation (2).

Figure 2 shows the calculated phase contrast over a sample with a buried point defect at depth $z_{0}$, taking into account only deviations of thermal conductivity. The curve parameter is $z_{\mathcal{O}} / \mu$, which is proportional to $f^{7 / 2}$ ( $f$ - modulation frequency). The contrast just above the defect increases with increasing $z_{\alpha} / \mu$ and reaches a first extreme at $z_{\alpha} / \mu=0.27$. With further increasing of the parameter $z_{\delta} / \mu$ the situation will be reversed. At $z_{d} / \mu=0.8$ a second contrast extreme of opposite sign appears. Enlarging the abscissa leads to decreasing and finally vanishing values of the contrast. At $z_{d} / \mu$ of about 0.57 the phase contrast curve shows a dip. as it would be expected in the case of two neighboured defects. This behaviour is due to the superposition of primarely generated and scattered thermal waves.

The amplitude contrast curves (figure 3 ) show a very similar behaviour, but with extremes at shifted parameters. So the amplitude contrast vanishes at $z_{\alpha} / \mu=1.44$ and shows a contrast maximum at $z_{o} / \mu=0.66$. Furthermore, it is easy to understand that for deeper defects the contrast curve decreases and its shape broadens.

The phase and amplitude contrast functions calculated for two neighboured defects with same defect strength, but different depthes $z_{0}$ and $z_{1}$ are shown in figure 4 and 5 , respectively. The curves were calculated in dependence of modulation frequency $f$, the parameter is $z / \mu$ $(i=0,1)$.

The variation of the modulation frequency changes the photothermal image. Despite the different depth combinations of $z / \mu$ exist, where the deeper defect seems to have a higher defect strength - and/or - seems to be located nearer to the surface (curve a in figure 4) or for another $z / \mu$ the defects seem to be similar in depth and strength (curve $c$ in figure 4 ). In curve $d$ of figure 4 the thermal waves nearly reach the upper defect $\left(z_{\alpha} / \mu \approx 1\right)$, phase contrast image shows the defect very clear and stronger than the deeper defect. For an accurate interpretation of photothermal images it is therefore necessary to compare both phase and amplitude contrast images. In the case of vanishing phase contrast it is still possible to obtain informations about the defect from amplitude contrast (see curve $b$ in figure 4 and curve $b$ in figure 5), caused by the different frequency behaviour of phase and amplitude contrast.

In order to achieve highest contrast and decent signal-to-noise ratio we have to choose an appropriate low modulation frequency corresponding to an extreme of the contrast functions. 


\section{http://dx.doi.org/10.21611/qirt.1992.039}

\section{Estimation of the resolution power}

The resolution of photothermal imaging can be quantified in terms of the halfwidth at half contrast (HWHC). It was shown, that for $z_{\alpha} / \mu<0.2$ the HWHC depends strongly on the defect depth but it is nearly independent on modulation frequency [8]. To summarize a body of numerical calculations of contrast which are stated in [9] we conclude:

- the contrast of the $k$-defect is higher than that of a $(\varrho \cdot c)$-defect;

- neighboured $k$-defects can be resolved better than $(\varrho \cdot c)$-defects;

- in the near field limit two buried $k$ - or $(\varrho \cdot c)$-defects can be distinguished if their lateral distance $x_{0}$ exceeds $1.5 z_{0}$ or $2 z_{0}$, respectively;

- for non zero heating beam radius $R, z_{o}$ has to be replaced by $\left(R^{2}+z_{0}^{2}\right)^{1 / 2}$ in the resolution condition stated above.

\section{Experimental}

To illustrate the essential features of the contrast considerations we experimentally investigated an appropriately designed model sample. Tiny grooves had been engraved into the surface of a plane brass sample. The depth and width of the grooves are $480 \mu \mathrm{m}$ and $950 \mu \mathrm{m}$ or $250 \mu \mathrm{m}$ and $300 \mu \mathrm{m}$, respectively. The double grooves were separated by $1200 \mu \mathrm{m}$ or $100 \mu \mathrm{m}$, respectively. Then the whole surface was uniformely tinned and finally polished. The mean thickness of the tin overcoating was about $300 \mu \mathrm{m}$. The finished sample surface was photothermally scanned, using two different techniques. We used the PTM1, a radiometric microscope made by Phototherm Dr Petry $\mathrm{GmbH}$, Saarbrücken, and a Mirage interferometer as already reported in $[10$ and 11$]$. Measured phase scans (figure 6) performed at appropriately low frequencies reproduced the brass profile but additionally showed a reproducable fine structure resulting from the brass roughness and tin crystallization.

A more detailed scan of the surrounding of the groove labeled 'position 2' in figure 6 was done by Mirage interferometry. From these curves the phase contrast at various modulation frequencies was estimated. The results obtained both from radiometric and Mirage interferometric measurements are given in figure 7. Both curves coincide fairly well. As it was predicted above there exist a contrast maximum and a zero crossing somewhere around the double abscissa value. We have to mention that the absolute abscissa of the measured contrast maximum does not agree exactly with the calculated one. This fact is believed to be caused by some uncertainty of the thermal tin parameters and by some deviations of the brass-tin sample from the theoretical model.

\section{Summary}

To describe photothermal imaging we introduced a point spread function (PSF) which is defined by the photothermal image of a buried point-like distortion of the thermal sample properties. The PSF allows us to estimate the photothermal signal in the presence of any excitation and defect distribution. To calculate the PSF we had to solve the inhomogeneous heat diffusion equation using a pertubation-theoretical technique. For appropriate interpreting photothermal scanning measurements amplitude and phase contrast functions could be deduced from the PSF.

These functions show extrema and zero crossings at certain frequencies. So we expect the maximum phase contrast of a single $k$-defect at a modulation frequency with $z_{o}=0.3 \mu$. For two buried thermal defects one gets a resolution criterium as follows: the $k(\varrho \cdot c)$-defects located at depth $z_{o}$ can be resolved, if their lateral distance is $x_{0} \geq 1.5 z_{0}\left(x_{0} \geq 2 z_{0}\right)$.

To illustrate the presented theoretical results we measured the phase contrast scanning a appropriately designed brass sample in dependence on modulation frequency using photothermal radiometry as well as mirage interferometry. These measurements coincide in a fairly good manner with our theoretical forecast. 


\section{Acknowledgements}

The authors are indebted to K.Friedrich, K. Haupt, G.Busse and B.K.Bein for brainstorming discussions and useful advises. Experimental work was friendly supported by Phototherm $\mathrm{Dr}$ Petry $\mathrm{GmbH}$, Saarbrücken.

\section{REFERENCES}

[1] BUSSE (G.) and ROSENCWAIG (A.). - Subsurface imaging with photoacoustics. Appl.Phys.Lett. 36, 1980, p. 815.

[2] BUSSE (G.) and WALTHER (H.G.). - in: Progress in Photothermal and Photoacoustic Sciences and Technology. Vol. 1, ed. by A.Mandelis, Elsevier Appl. Science, London and NY., (1991).

[3] MANDELIS (A.), J.Opt.Soc.Am. A, 6, 1989, p. 298.

[4] MUNIDASA (M.) and MANDELIS (A.). - Same as [2].

[5] WETSEL (G.C.) and MCDONALD (F.A.). - Resolution and definition in photothermal imaging. J.Appl.Phys. 56, 1981, p. 3081.

[6] BORN (M.) and WOLF (E.). - Principles of optics, Pergamon Press Oxford, 1989.

[7] WALTHER (H.G.), SEIDEL (U.), KARPEN (W.) and BUSSE (G.). -Application of modulated photothermal radiometry to infrared transparent samples. Submitted to Rev.Sci.Instr.

[8] FRIEDRICH (K.), HAUPT (K.), SEIDEL (U.) and WALTHER (H.G.). - Some remarks on definition, resolution and contrast in photothermal imaging, will be published in J.Appl. Physics .

[9] FRIEDRICH (K.). - Beiträge zur photothermischen Mikroskopie an Festkörpern. Ph.D. Thesis, Jena, 1991.

[10] WALTHER (H.G.), FRIEDRICH (K.), GÖRING (R.), HAUPT (K.) and SEIDEL (U.). - in: Proceedings of the 7 th Int. Topical Meeting on Photoacoustic and Photothermal Phenomena. Bicanic (D.) ed., Doorweerth (The Netherlands), 1991, p. 292.

[11] WALTHER (H.G.), FRIEDRICH (K.), HAUPT (K.), MURATIKOV (K.L.) and GLAZOV (A.L.). New phase interference technique applied for sensitive photothermal microscopy. Appl.Phys.Lett.57, 1990, p. 1600.

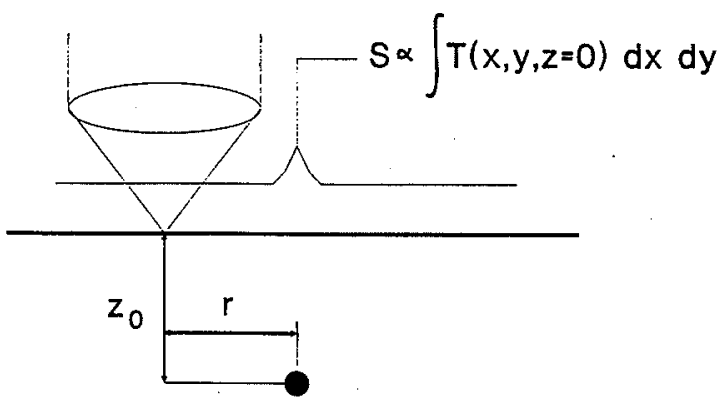

Fig.1. - Scheme of photothermal imaging of a thermal point defect using point-like heating and large area detector; $(r$ - lateral distance between defect and excitation, $z_{0}$ - defect depth) 
http://dx.doi.org/10.21611/qirt.1992.039

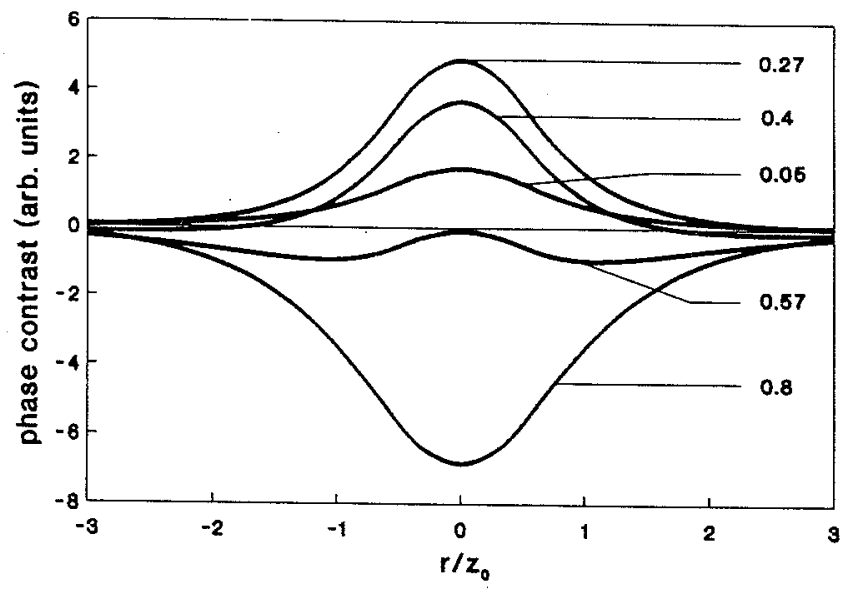

Fig.2. - Phase contrast of a subsurface $k$-defect at a constant depth $z_{0}$ versus normalized distance between defect and excitation spot $r / z_{0}$ (parameter is $z d \mu$ )

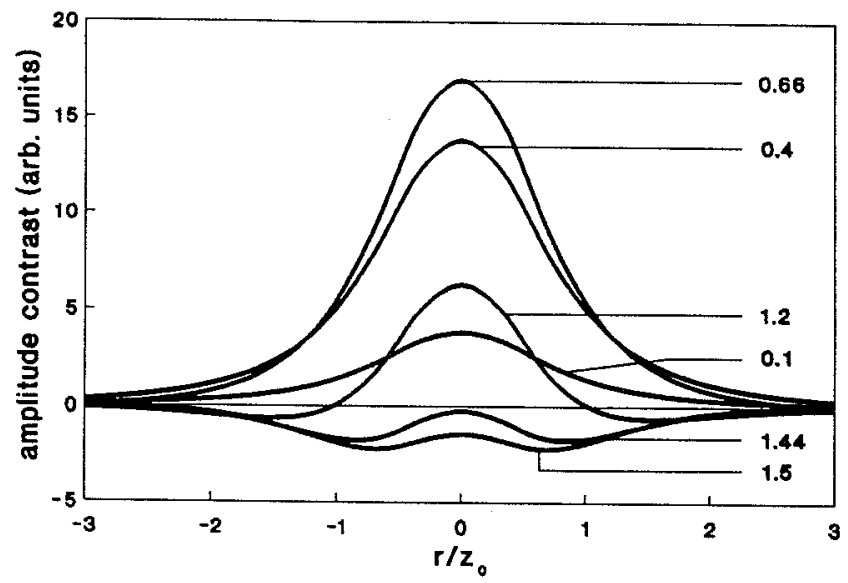

Fig.3. - Amplitude contrast of a subsurface $k$-defect (according to figure 2) 


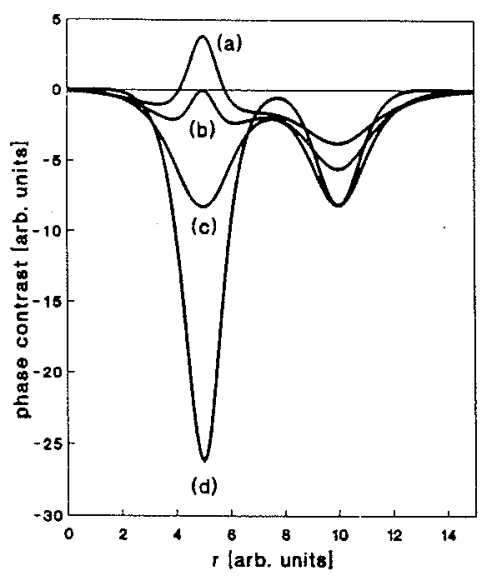

Fig.4. - Phase contrast of two $k$-defects at different depthes $\quad z_{\sqrt{ }} / z_{0}=1.5 \quad\left(a-z_{\delta} / \mu=0.5 ; \quad b\right.$ $\left.z_{d} / \mu=0.57 ; \mathrm{c}-z_{d} / \mu=0.69 ; \mathrm{d}-z_{d} / \mu=1\right)$

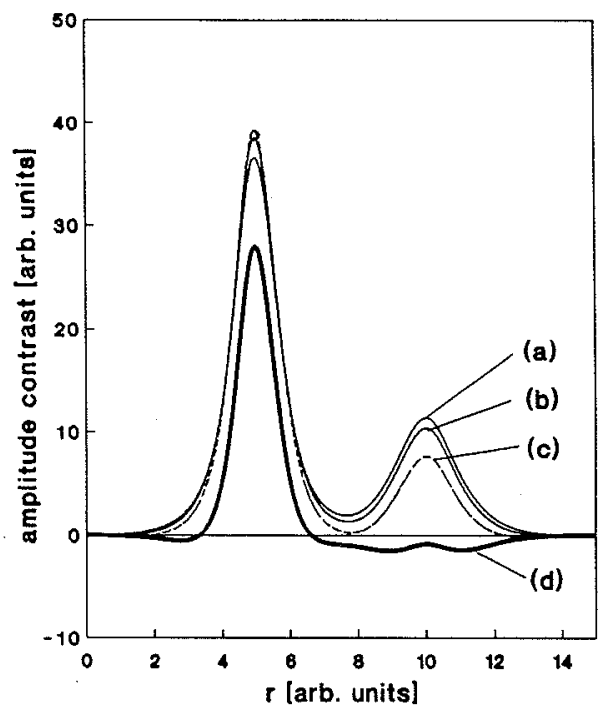

Fig.5. - Amplitude contrast of two $k$-defects (according to figure 4) 


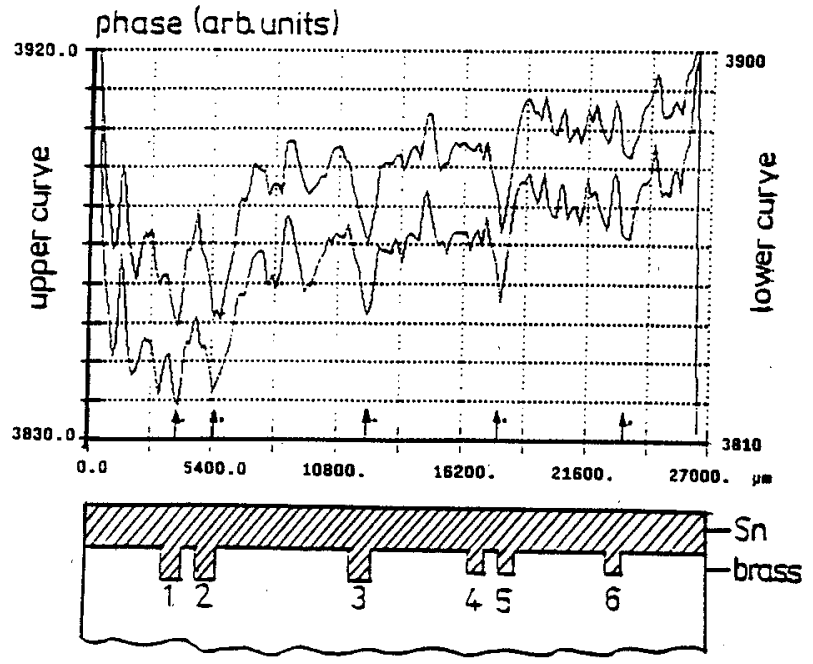

Fig.6. - Measured phase scans over the tinned brass sample (as sketched below), (upper curve: $40 \mathrm{~Hz}$, lower curve: $60 \mathrm{~Hz}$ ); the closely neighboured grooves 4 and 5 couldn't be resolved (photothermal radiometry, PTM1)

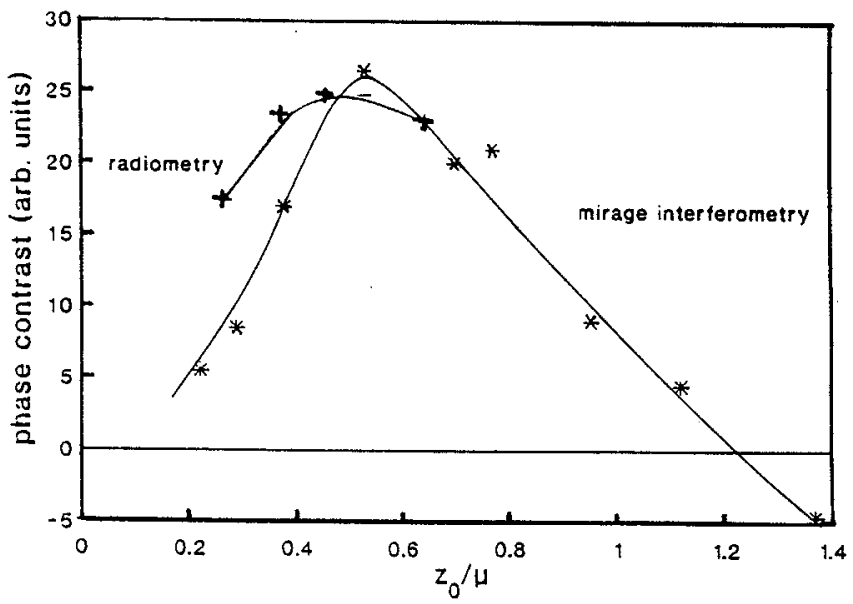

Fig.7. - Phase contrast versus $z_{d} \boldsymbol{\mu}$, estimated from radiometric and mirage-interferometric measurements 